Tecnociencia, Vol. 23, $\mathrm{N}^{\circ} 1: 82-103$

Enero-Junio 2021

\title{
COLONIZACIÓN DE LA HEPÁTICA EPÍFILA Leptolejeunea elliptica (LEHM \& LINDENB) SCHIFFN. (LEJEUNEACEAE), SOBRE DOS ESPECIES DE ARBUSTOS DE LA ISLA BARRO COLORADO (BCI)
}

\author{
${ }^{1}$ Lilisbeth Rodríguez-Castro (iD) $\mathbf{y}^{\mathbf{2}}$ Nayda Flores \\ ${ }^{1}$ Universidad de Panamá, Facultad de Ciencias Naturales, Exactas y Tecnología, \\ Escuela de Biología. E-mail: lili_0990@ outlook.es \\ ${ }^{2}$ Universidad de Panamá, Facultad de Ciencias Naturales, Exactas y Tecnología, \\ Departamento de Botánica. E-mail: naydaf@gmail.com
}

\section{RESUMEN}

La tasa de crecimiento de las comunidades de briofitas epífitas está ligeramente correlacionada con la humedad atmosférica, pero la apertura del dosel (luz), temperatura y precipitación, también representan factores ambientales importantes en su crecimiento. En este trabajo se estudió la colonización de la especie epifila Leptolejeunea elliptica (Lehm \& Lindenb) Schiffn. sobre hojas de Hybanthus prunifolius y Psychotria horizontalis en cuatro micrositios de la Isla Barro Colorado, tomando en cuenta factores como la luz, temperatura y humedad relativa. Se seleccionó un sitio húmedo y un sitio seco, cada sitio contaba con claros y sombras; teniendo en total cuatro micrositios. En cada micrositio, se seleccionaron cinco individuos de cada especie de arbusto y de cada uno, se seleccionaron cinco hojas. Cada hoja fue monitoreada cada dos semanas por un periodo de 6 meses. En cada hoja se observó la cobertura y colonización de L. elliptica. Además, se midió la humedad y temperatura en cada sitio. Valores de humedad y temperatura obtenidos nos mostraron que todos los micrositios se encontraban en condiciones similares, por lo que concluimos que estos factores no afectaron la cobertura y colonización de L. elliptica. El factor más determinante fue la luz, lo que demuestra que esta hepática es 
común en claros y es una especie indicadora de perturbaciones en los bosques tropicales.

\title{
PALABRAS CLAVES
}

Leptolejeunea elliptica, hepática, epifila, Isla Barro Colorado.

\section{COLONIZATION OF THE EPIPHYLLOUS LIVERWORT LEPTOLEJEUNEA ELLIPTICA (LEHM \& LINDENB) SCHIFFN. (LEJEUNEACEAE) ON TWO SHRUBS SPECIES FROM BARRO COLORADO ISLAND (BCI)}

\begin{abstract}
The growth rate of epiphyllous bryophytes communities is slightly correlated with atmospheric humidity, however canopy opening (light), temperature, and precipitation represent important environmental factors in their growth. In this work we studied the colonization of the epiphyllous species Leptolejeunea elliptica (Lehm \& Lindenb) Schiffn., growing on leaves of Hybanthus prunifolius and Psychotria horizontalis in four microsites on Barro Colorado Island, considering factors such as light, temperature and relative humidity. We selected a wet site and a dry site, each site had gaps and shadows, having in total four microsites. In each microsite five plants of each species of shrubs were selected and from each one five leaves were selected. Each leaf was monitored every two weeks for a period of 6 months. On each leaf, the coverage and colonization of L. elliptica was observed. In addition, humidity and temperature were measured at each site. The values of humidity and temperature showed that all microsites were in similar conditions, so we concluded that these factors did not affect the coverage and colonization of L. elliptica. The factor that had the greatest significance was light, which show that L. elliptica is a common species in gaps and an indicator species of disturbances in tropical forests.
\end{abstract}

\section{KEYWORDS}

Leptolejeunea elliptica, liverwort, epiphyllous, Barro Colorado Island.

\section{INTRODUCCIÓN}

Una de las características más llamativas en los bosques húmedos tropicales es la abundancia de plantas epífilas (Pócs, 1982; Sonnleitner et al., 2009). La comunidad de epífilas está compuesta de especies de diferentes grupos taxonómicos como hepáticas, musgos, líquenes, algas, 
cianobacterias, hongos e incluso de algunos animales microscópicos (Ruinen, 1961). En cuanto a briofitas epífilas en sentido amplio, la diversidad taxonómica está restringida principalmente a tres familias, la familia de hepáticas Lejeunaceae que representan el grupo más diverso con más del 95\% de todas las epífilas, la Radulaceae y los musgos de la familia Hookeraceae (Gradstein, 1997; Sonnleitner et al., 2009).

Debido a su condición poiquilohídrica, las briofitas dependen del microclima en donde se desarrollan (Winkler, 1967; Olarinmoye, 1974; Marino \& Salazar Allen, 1993; Lücking, 1995; Coley \& Kursar, 1996; Sonnleitner et al., 2009). Algunos autores sugieren que los factores microclimáticos controlan la distribución, abundancia, expresión reproductiva y crecimiento de las epifilas (Richards, 1954; Pócs, 1982; Marino \& Salazar Allen, 1993). Por otro lado, numerosos estudios de distribución no cuantitativos muestran que las hepáticas están asociadas a sitios muy húmedos (Smith, 1982; Pocs, 1982), siendo la humedad relativa el factor principal que determina la estructura de las comunidades epifilas (Winkler, 1967; Olarinmoye, 1974; Marino \& Salazar Allen, 1993; Lücking, 1995; Coley \& Kursar, 1996; Sonnleitner et al., 2009). Sin embargo, características de las especies de plantas hospederas como la textura y el tiempo de vida de las hojas, la variación asociada a la ubicación en el dosel y la ubicación en relación con los claros de luz, también pueden tener influenciar en la estructura y composición de la comunidad de epífilas (Marino \& Salazar Allen, 1993).

Marino \& Salazar Allen (1993), demostraron que el establecimiento de las comunidades epífilas sobre especies de arbustos del sotobosque de la isla de Barro Colorado, fueron fuertemente influenciadas por la cantidad de luz y las características del micrositio. Sus resultados muestran que Leptolejeunea elliptica domina en los sitios húmedos, y es rara en los sitios más secos. En este mismo sentido, estudios realizados por Zartman et al. (2015) en el Amazonas durante la época seca, demostraron que las comunidades de briofitas tuvieron una reducción en el crecimiento de sus colonias, disminución de supervivencia y reducción en la expresión asexual y sexual. Por lo tanto, las variaciones en el micrositio como la luz y la humedad podrían modular la colonización y reproducción sexual o asexual de las especies de briofitas epífilas (Coley \& Kursar, 1996; Maciel-Silva et al., 2012). 
Zartman et al. (2012), mencionan que los eventos de colonización de epífilas están determinados por la conectividad a corta distancia entre las comunidades a nivel local, donde estas muestran dispersión limitada; refiriéndose con evento de colonización al momento en el que la epífila coloniza por primera vez la hoja. Sin embargo, Marino (1991) indicó que la estructura y diversidad de las comunidades de briofitas que ocurren sobre excremento (Splachnaceae), están determinadas por las interacciones interespecíficas, las condiciones microclimáticas y la competitividad entre diferentes especies. Se ha sugerido que la composición y la diversidad de las comunidades de briofitas en diferentes hábitats están limitadas, principalmente, por la capacidad de dispersión y la tolerancia fisiológica de las especies (Slack, 1977).

Este trabajo tiene como objetivo el estudio de la colonización y cobertura de la hepática foliosa Leptolejeunea elliptica tomando en cuenta factores microclimáticos que determinan la estructura de estas comunidades, para eso se establecerá la relación entre el efecto del micrositio con el momento de colonización y la cobertura de comunidades de L. elliptica. Este tipo de estudio demuestra la importancia de las briofitas como indicadoras de perturbaciones en los ecosistemas tropicales naturales.

\section{MATERIALES Y MÉTODOS}

1. Área de Estudio

El estudio se realizó en el monumento Natural Barro Colorado, localizado en el Lago Gatún $\left(9^{\circ} 09^{\prime} \mathrm{N} 79^{\circ} 51^{\prime} \mathrm{O}\right)$, en el centro del Canal de Panamá (Fig. 1). Está conformado por las penínsulas de Bohío, Buena Vista, Peña Blanca, Gigante, Frijoles y la Isla Barro Colorado (BCI, por sus siglas en inglés), abarcando en su conjunto un área total de 5364 hectáreas, de éstas, 1564 pertenecen a la Isla Barro Colorado. Su punto más alto se encuentra a 145 m.s.n.m (Wong \& Ventocilla, 1987). El promedio de humedad relativa permanece alto todo el año, con valores mínimos de 62 a $68 \%$ en abril, y máximos de 80 a $100 \%$ en noviembre (Dietrich et al., 1992), el promedio anual de temperatura es de $27^{\circ} \mathrm{C}$. Según la clasificación de zonas de vida de Holdridge, la Isla Barro Colorado está situada en la zona de bosque húmedo tropical; según el sistema de clasificación de climas de Köppen, posee un clima 
tropical húmedo con una precipitación pluvial anual de $2641 \mathrm{~mm}$ (Claver, 1984), y según el mapa de vegetación posee bosque semicaducifolio tropical de tierras bajas (ANAM, 2000).

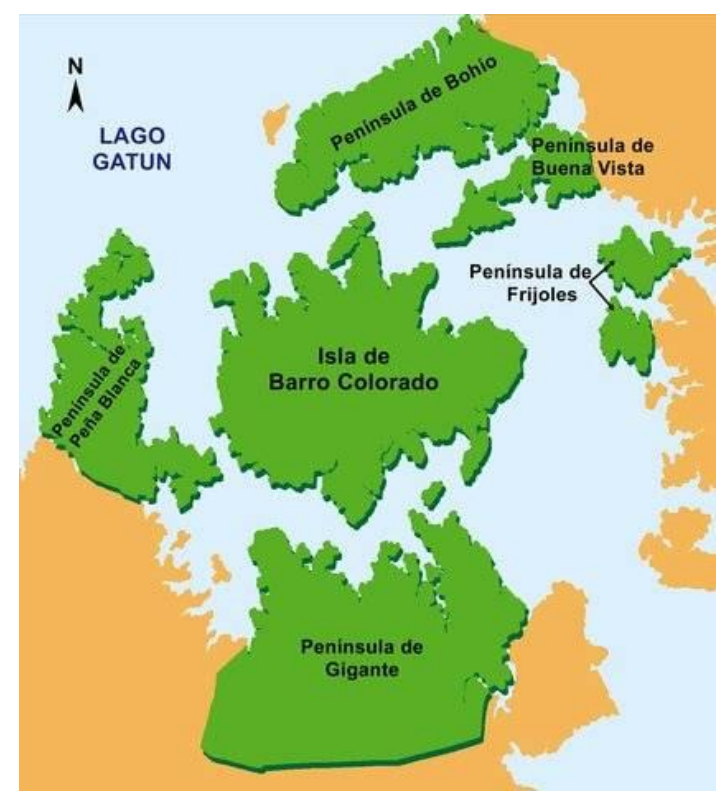

Fig. 1 Ubicación geográfica del Monumento Natural Barro Colorado

2. Descripción de la especie de estudio

Leptolejeunea elliptica (Lehm \& Lindenb) Schiffn es una especie de hepática epífila común en la región tropical. El género Leptolejeunea es casi exclusivamente epífilo (Pócs, 1996). Leptolejeunea elliptica es frecuente en los bosques húmedos de tierras bajas (menos de $500 \mathrm{~m}$ ), sobre hojas, y rara vez sobre corteza (Gradstein \& Ilkiu Borges, 2009), no es exclusiva de bosque primario. Se seleccionó L. elliptica, por ser una especie común sobre las hojas de arbustos del sotobosque en BCI, la misma presenta hojas patentes que al estar secas se elevan del sustrato. También tiene un olor característico a menta, debido a la cantidad de terpenoides (Toyota et al., 1997). Esta característica ayuda a identificarla en campo, sin necesidad de colectas, lo que permite que sea monitoreada a lo largo del tiempo sin perturbar las poblaciones.

3. Descripción de las plantas hospederas

Para este estudio se utilizaron las hojas de Hybanthus prunifolius (Humb. \& Bonpl. ex Schult.) Schulze-Menz y Psychotria horizontalis 
Sw., dos especies de arbustos muy comunes en BCI. Hybanthus prunifolius es un arbusto que puede llegar a medir hasta $7 \mathrm{~m}$ de altura, presenta hojas alternas, elípticas, $4.5-15 \mathrm{~cm}$ de largo, atenuadas a acuminadas en el ápice, lisas, adaxialmente seríceas a lo largo del nervio principal, abaxialmente seríceas cuando jóvenes, tricomas persistentes en la base y a lo largo del nervio principal; tiene ramas jóvenes pilosas, estriadas y grises claros. Psychotria horizontalis es un arbusto que puede alcanzar hasta los $3 \mathrm{~m}$ de alto, presenta hojas opuestas, elípticas a oblanceoladas, de 3 a $9 \mathrm{~cm}$ de largo y $1.5 \mathrm{a} 4.5 \mathrm{~cm}$ de ancho, el ápice es agudo a ligeramente acuminado, margen entero y presenta domacios en la unión entre las venas secundarias con la primaria. Ambas especies de arbustos tienen una vida media de 1 año, las hojas las pierden en la estación seca, de enero a abril (Aide, 1989).

\section{Muestreo}

4.1 Fecha de estudio y selección del micrositio

Este estudio se efectuó durante los meses de agosto de 2016 hasta febrero de 2017, contemplando parte de la época lluviosa, que va de abril a noviembre, e inicios de la época seca que va desde diciembre a marzo. Se eligió el sendero Barbour Lathrop porque tiene quebradas, por lo cual se consideró el micrositio más húmedo, además tiene partes más altas con condiciones de menor humedad que sirvió para el micrositio menos húmedo. También contaba con la población necesaria de los arbustos hospederos. Luego de haber seleccionado los micrositios, se registró la humedad y temperatura en cada uno de ellos. Para esto se colocó en cada sitio, al mismo tiempo, un registrador de datos (sensor de humedad y termómetro electrónico) Logging Moment Values, número de serie 080111521217, a una altura de $1.30 \mathrm{~m}$ del suelo; que tomó datos durante 48 horas cada 32 minutos. Esta toma de datos se realizó una vez para la época lluviosa (noviembre) y otra en la época seca (febrero). De igual manera, se tomaron en cuenta datos de precipitación durante esos meses, facilitados por el Programa de monitoreo físico de Smithsonian Tropical Research Institute. La apertura del dosel e intensidad de luz fue medida con la aplicación móvil Gap Light Analysis. 
4.2 Selección de las plantas hospederas y las hojas

En cada micrositio, se seleccionaron cinco plantas hospederas, haciendo un total de 40 plantas, 20 en el sitio húmedo (diez en claro y diez en sombra) y 20 en el sitio menos húmedo (diez en claro y diez en sombra). En cada arbusto se seleccionaron y marcaron cinco hojas jóvenes aún no colonizadas por Leptolejeunea elliptica, haciendo un total de 200 hojas. Las mismas fueron monitoreadas cada dos semanas durante un periodo de seis meses; la colonización fue determinada como el momento en que las primeras ramas de L. elliptica se establecieron sobre las hojas monitoreadas, para ello se contó el número de ramas que se encontraban creciendo sobre las hojas de los arbustos. Al mismo tiempo, se cuantificó el crecimiento en función al área de cobertura de la epífila; para esto se utilizó papel de filmina cuadriculado en $\mathrm{mm}^{2}$, en las cuales se hizo el cálculo de la cobertura, este mismo papel de filmina fue utilizado para estimar el área total de la hoja.

\section{Análisis de datos}

Para el análisis de los datos se utilizó el programa $\mathrm{R}$ stadistical programming language ( $\mathrm{R}$ Core Team). Se realizó la prueba de Wilcoxon $(\alpha=0.05)$ para comparar la cobertura de L. elliptica en cada arbusto con cada una de las variables microclimáticas y determinar cuál de ellas tuvo mayor influencia sobre la cobertura de Leptolejeunea elliptica. También se realizó un estadístico de modelo lineal generalizado donde se muestran los factores con mayor influencia en la cobertura.

\section{RESULTADOS Y DISCUSIÓN}

\section{Colonización}

Se determinó la cantidad promedio de ramas que colonizaban por primera vez (Cuadro 1). En cada conteo se tomaron en cuenta las 25 hojas (cinco hojas de cada arbusto) en cada uno de los cuatro micrositios; luego se tomó el promedio de ramas de L. elliptica que había en cada una de las hojas que se encontraban en el mismo sitio y en la misma especie de arbusto.

En el primer censo todos los valores fueron cero, ya que todas las hojas seleccionadas eran jóvenes y no colonizadas. Desde el censo 2 en adelante se muestra la cantidad promedio de nuevas ramas que habían colonizado las hojas en cada micrositio (Cuadro 1).

Los valores mostrados en el Cuadro 1 no son sumativos, estos indican la cantidad de nuevos individuos que colonizaron la hoja para cada 
censo, por ejemplo, los individuos contabilizados en el censo 3, no son los mismos contabilizados antes en el censo 2 y así sucesivamente. Cada conteo se realizó en la misma hoja seleccionada desde el primer día.

Cuadro 1. Número de ramas de L. elliptica que colonizaron las hojas de H. prunifolius y $P$. horizontalis.

\begin{tabular}{|c|c|c|c|c|c|c|c|c|c|c|}
\hline \multirow{2}{*}{ Micrositio } & \multicolumn{9}{|c|}{ Censo (fecha) } & Total \\
\cline { 2 - 12 } & $\mathbf{1}$ & $\mathbf{2}$ & $\mathbf{3}$ & $\mathbf{4}$ & $\mathbf{5}$ & $\mathbf{6}$ & $\mathbf{7}$ & $\mathbf{8}$ & $\mathbf{9}$ & \\
& $(24 / 08$ & $(14 / 0$ & $(05 / 1$ & $(26 / 10 /$ & $(16 / 1$ & $(07 / 1$ & $(28 / 1$ & $(18 / 0$ & $(08 / 0$ & \\
\hline HPSL & 0 & 7 & 7 & 4 & 0 & 1 & 0 & 0 & 1 & 20 \\
\hline HPHL & 0 & 8 & 4 & 2 & 1 & 2 & 0 & 0 & 1 & 18 \\
\hline HPSS & 0 & 0 & 3 & 1 & 0 & 2 & 0 & 1 & 3 & 10 \\
\hline HPHS & 0 & 2 & 3 & 0 & 3 & 1 & 0 & 0 & 1 & 10 \\
\hline PHSL & 0 & 5 & 2 & 2 & 0 & 1 & 0 & 0 & 2 & 12 \\
\hline PHHL & 0 & 10 & 3 & 1 & 0 & 0 & 0 & 0 & 0 & 14 \\
\hline PHSS & 0 & 0 & 5 & 1 & 0 & 0 & 0 & 0 & 0 & 6 \\
\hline PHHS & 0 & 5 & 4 & 0 & 2 & 0 & 0 & 0 & 1 & 12 \\
\hline
\end{tabular}

HPSL $=H$. prunifolius seco con luz. HPHL $=H$. prunifolius húmedo con luz. HPSS= $H$. prunifolius seco con sombra. $\mathrm{HPHS}=H$. prunifolius húmedo con sombra. $\mathrm{PHSL}=$ $P$. horizontalis seco con luz. $\mathrm{PHHL}=P$. horizontalis húmedo con luz. $\mathrm{PHSS}=P$. horizontalis seco con sombra. PHHS= P. horizontalis húmedo con sombra.

A partir de los datos mostrados en el Cuadro 1, se construyeron las gráficas de las Figuras 2 y 3, buscando presentar de manera mucho más clara los meses en los que se observó mayor colonización de L. elliptica en los respectivos arbustos y micrositios. A pesar de que no existió una alta diferencia entre el número de colonizaciones para cada micrositio, se puede observar como en el censo 2, realizado en el mes de septiembre, hubo mayor cantidad de ramas colonizando las hojas de $P$. horizontalis ubicadas en el sitio húmedo y con luz (Figura 3B), seguido de las hojas en el mismo micrositio, pero en $H$. prunifolius (Figura 2B), coincidiendo con lo mencionado por Marino \& Salazar (1993), de que L. elliptica es una epífila común de lugares muy húmedos y con alta intensidad de luz. El valor que le sigue a los dos anteriores es el del sitio seco y luz, con una colonización de 7 ramas promedio en los censos 2 y 3 (Figura 2A), esto puede deberse a que las diferencias entre el sitio húmedo y el sitio seco no fueron tan grandes en cuanto a la humedad (Cuadro 3), pero un 
factor en común que tienen estos tres micrositios donde se registró la mayor colonización promedio son los claros. Monge-Najera \& Blanco (1995) mencionan que existe la posibilidad de que los propágulos lleguen a las hojas más fácilmente en los sitios claros, esto podría deberse a que en los claros las hojas se encuentras más expuestas y por ende tendrán mayor oportunidad de ser colonizadas en comparación con aquellas que están cubiertas, pero esto aún queda por ser comprobado. De igual forma al observar el total de colonizaciones por micrositio, se observa cómo fueron las hojas de $H$. prunifolius en el sitio seco con luz y sitio húmedo con luz los que tuvieron mayor porcentaje de colonización total de L. elliptica.
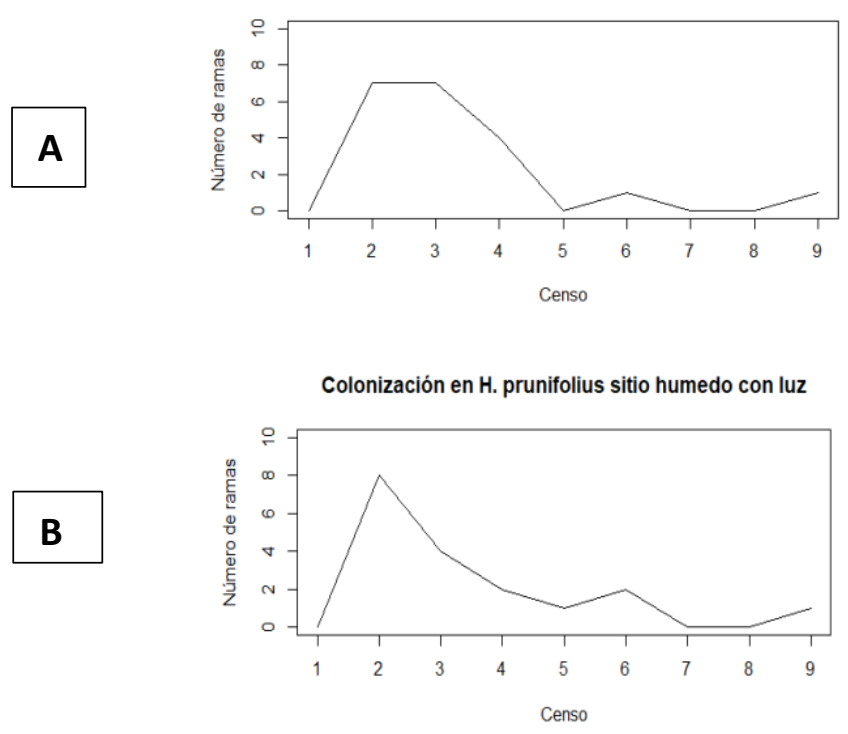

Castro. R. L\& Flores, $N$. 

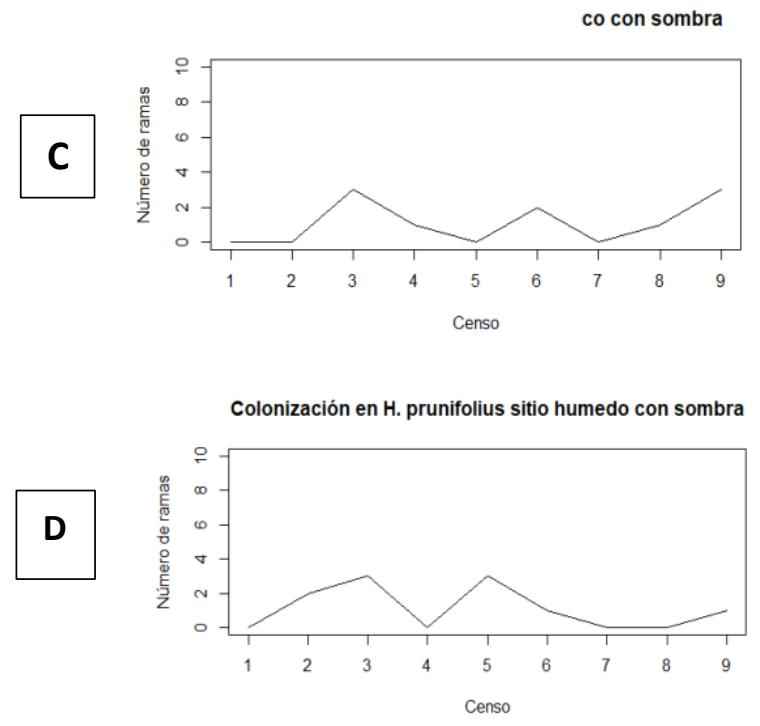

Fig. 2 Colonización de L. elliptica en hojas de H. prunifolius en los cuatro micrositios. A. Colonización en $H$. prunifolius sitio seco con luz. B. Colonización en H. prunifolius sitio húmedo con luz. C. Colonización en $H$. prunifolius sitio seco con sombra. D. Colonización en $H$. prunifolius sitio húmedo con sombra.

En las gráficas de la Figura 2, no se observa ningún patrón de colonización de L. elliptica, aunque si se observa una colonización mayor en las hojas ubicadas en sitios con luz en comparación con los sitios ubicados en sombra. En los censos 7 y 8 se observó una gran disminución de la colonización para todos los micrositios, posiblemente porque estos censos se realizaron entre los meses de diciembre y enero, en los cuales comienza la estación seca y los arbustos comienzan a perder muchas hojas. 

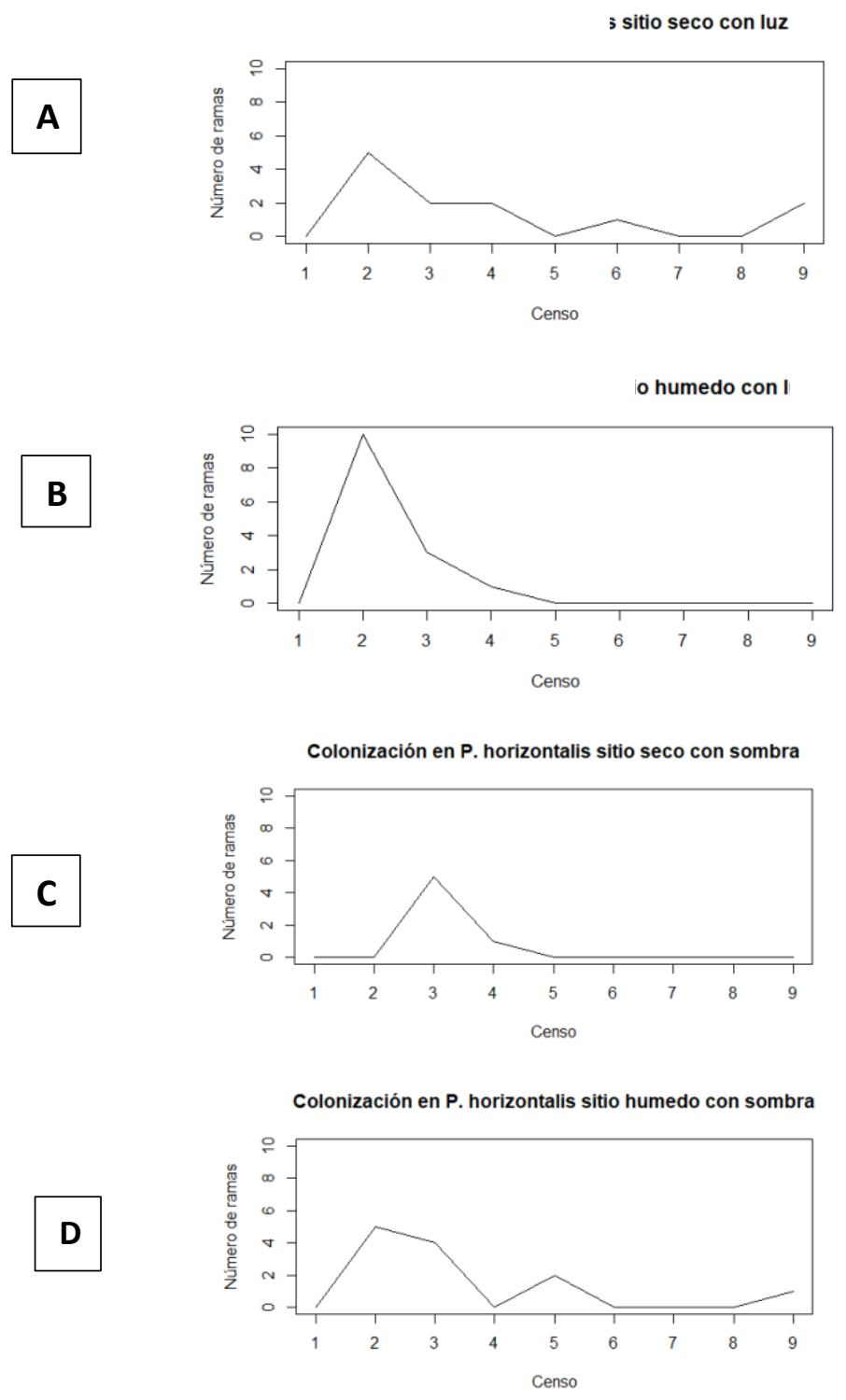

Fig. 3 Colonización de L. elliptica en hojas de $P$. horizontalis en los cuatro micrositios. A. Colonización en $P$. horizontalis sitio seco con luz. B. Colonización en $P$. horizontalis sitio húmedo con luz. C. Colonización en $P$. horizontalis sitio seco con sombra. D. Colonización en $P$. horizontalis sitio húmedo con sombra.

Utilizando los datos de precipitación durante los meses de estudio, se construyeron las gráficas de la Figura 4 en donde se observa un aumento 
en la precipitación durante el mes de noviembre. Diversos autores (Olarinmoye, 1975; Richards, 1984; Coley et al., 1993) mencionan que a pesar de que hay mucha evidencia cualitativa que sugiere que las hepáticas epífilas requieren una proporción alta de agua, la lluvia intensa directa parece impedir el desarrollo de estas. En las Figuras 2A, 2B, 2C, 3A, 3B y 3C se observa una disminución en la colonización en el censo 5 (Cuadro 1) que se realizó durante el mes de noviembre y coincide con el aumento de la precipitación, esto puede deberse a que a los propágulos se les dificulta mucho más poder adherirse a las hojas cuando se encuentran mojadas, o si logran hacerlo la lluvia lava la hoja impidiendo que la briofita la colonice.

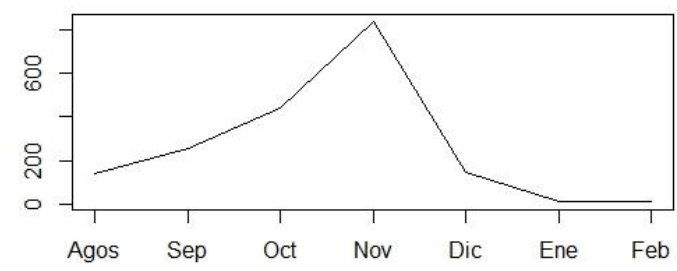

Fig. 4 Precipitación en mm para BCI (Programa de Monitoreo Físico, 2016 y 2017, STRI).

\section{Cobertura}

En el Cuadro 2 se muestra el porcentaje promedio de cobertura de $L$. elliptica en las 25 hojas (cinco hojas para cada arbusto) en cada uno de los micrositios. Estos valores indican qué tanto fue cubriendo L. elliptica durante cada uno de los censos; de igual manera se observa como la cobertura fue disminuyendo en algunos casos. Al igual que en el Cuadro 1 , los valores del primer censo son ceros debido a que no había cobertura de la hepática sobre ninguna de las hojas. En el Cuadro 2 también se observa que el censo 2 tiene la menor cobertura, esto se debe a que apenas estaba empezando la colonización de las hojas por la hepática $L$. elliptica. Por otro lado, el censo 9 realizado en el mes de febrero fue en el que se observó mayor cobertura. En el censo 7 también se observa como las hojas de $P$. horizontalis que se encontraban en el sitio húmedo con sombra tenían mayor cobertura que el resto, este censo se realizó 
finalizando el mes de diciembre, luego de haber pasado la época lluviosa, en donde las briofitas tenían suficiente humedad, pero sin fuertes lluvias que evitaran la adhesión de estas a la superficie de la hoja y suficiente luz para poder crecer.

Cuadro 2. Porcentaje de cobertura total de L. elliptica sobre las hojas de H. prunifolius y $P$. horizontalis.

\begin{tabular}{|l|l|l|l|l|l|l|l|l|l|}
\hline \multirow{2}{*}{$\begin{array}{l}\text { Micros } \\
\text { itio }\end{array}$} & \multicolumn{9}{|l|}{ Censo (fecha) } \\
\cline { 2 - 11 } & $\begin{array}{l}\mathbf{1}(24 / 08 / \\
16)\end{array}$ & $\begin{array}{l}\mathbf{2} \\
(14 / 09 / \\
16)\end{array}$ & $\begin{array}{l}\mathbf{3}(05 / 10 / \\
16)\end{array}$ & $\begin{array}{l}\mathbf{4}(26 / 10 / \\
16)\end{array}$ & $\begin{array}{l}\mathbf{5} \\
(16 / 11 / \\
16)\end{array}$ & $\begin{array}{l}\mathbf{6} \\
(07 / 12 / \\
16)\end{array}$ & $\begin{array}{l}\mathbf{7}(28 / 12 / \\
16)\end{array}$ & $\begin{array}{l}\mathbf{8} \\
(18 / 01 / \\
17)\end{array}$ & $\begin{array}{l}\mathbf{9} \\
(08 / 02 / \\
17)\end{array}$ \\
\hline HPSL & 0 & 1.5 & 3.6 & 3.4 & 3.3 & 6.7 & 6.7 & 4.2 & 6.2 \\
\hline HPHL & 0 & 1.7 & 3.1 & 4 & 4 & 3.4 & 3.3 & 4.4 & 5.6 \\
\hline HPSS & 0 & 0 & 0.1 & $0-2$ & 0.2 & 0.9 & 0.9 & 0.5 & 2.3 \\
\hline HPHS & 0 & 0.7 & 1.7 & 1.8 & 2.7 & 1.4 & 1.1 & 0.7 & 1.4 \\
\hline PHSL & 0 & 1 & 4 & 2.8 & 2.8 & 3.9 & 4 & 6.1 & 8.7 \\
\hline PHHL & 0 & 0.5 & 1.1 & 1.7 & 3.4 & 2.6 & 2.7 & 3.1 & 2.8 \\
\hline PHSS & 0 & 0 & 0.4 & 0.3 & 0.3 & 0.4 & 0.4 & 0.4 & 0.5 \\
\hline PHHS & 0 & 0.5 & 1.3 & 0.5 & 1 & 0.5 & 9.5 & 0.8 & 1.3 \\
\hline
\end{tabular}

HPSL $=H$. prunifolius seco con luz. HPHL $=H$. prunifolius húmedo con luz. HPSS= $H$. prunifolius seco con sombra. $\mathrm{HPHS}=H$. prunifolius húmedo con sombra. $\mathrm{PHSL}=$ $P$. horizontalis seco con luz. $\mathrm{PHHL}=P$. horizontalis húmedo con luz. $\mathrm{PHSS}=P$. horizontalis seco con sombra. $\mathrm{PHHS}=P$. horizontalis húmedo con sombra.

Utilizando los datos del Cuadro 2, se construyeron las gráficas de las Figuras 5 y 6 , en las que se muestra de manera más clara el porcentaje de cobertura de L. elliptica para cada micrositio para ambas especies de angiospermas. 


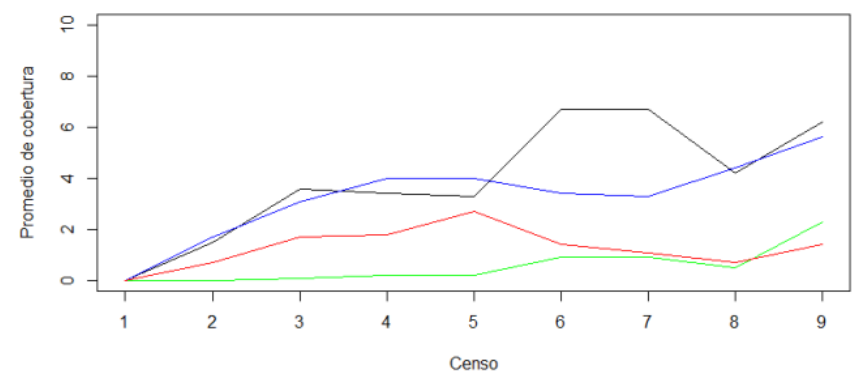

Negro $=$ sitio seco con luz. $\quad$ Azul= sitio húmedo con luz. Rojo= sitio húmedo con sombra. Verde $=$ sitio seco con sombra.

Fig. 5 Porcentaje promedio de cobertura de L. elliptica sobre hojas de $H$. prunifolius en los cuatro micrositios

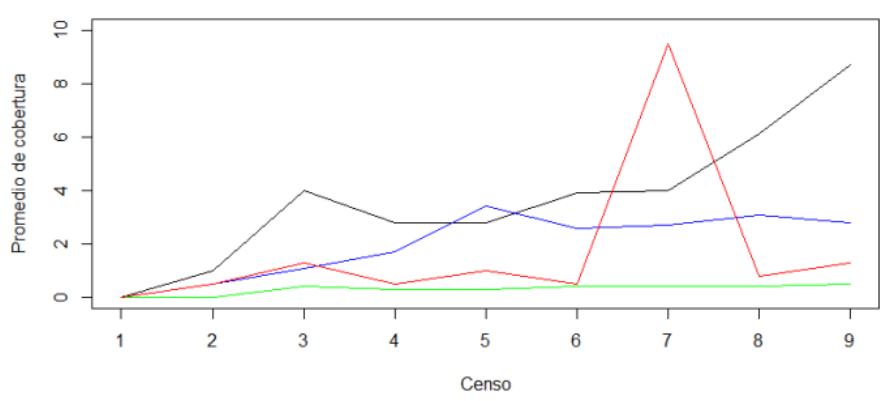

Negro $=$ sitio seco con luz. Azul= sitio húmedo con luz. Rojo $=$ sitio húmedo con sombra. Verde $=$ sitio seco con sombra.

Fig. 6. Porcentaje promedio de cobertura de L. elliptica sobre hojas de $P$. horizontalis en los cuatro micrositios

La cobertura en las hojas de cada uno de los arbustos para cada micrositio no sigue un patrón en especial, por ejemplo, las hojas de $H$. prunifolius en el sitio seco con sombra se comportan de manera distinta que las hojas de $P$. horizontalis para el mismo micrositio. 
Cuadro 3. Humedad relativa promedio para cada sitio

\begin{tabular}{|l|l|c|}
\hline Estación & Sitio & Humedad relativa \% \\
\hline Lluviosa & Húmedo & 97.3 \\
\hline Lluviosa & Menos húmedo & 94.1 \\
\hline Seca & Húmedo & 84.1 \\
\hline Seca & Menos húmedo & 83.5 \\
\hline
\end{tabular}

Siendo la humedad relativa uno de los principales factores que determinan la presencia o ausencia de comunidades epífilas, al realizar la prueba de Wilcoxon, se obtuvo un valor de

$\mathrm{p}=0.562(\mathrm{~W}=1016.5)$. Este valor demuestra que no hubo diferencia significativa en los porcentajes de cobertura respecto a la humedad, esto puede deberse a que los valores de humedad de los micrositios (Cuadro 3 ), no fueron muy diferentes como para provocar cambios en la cobertura. Autores como Fulford et al. (1970), Pocs (1982), Smith (1982), Richards (1984), Frahm (1987), Van Reenen (1987) y Thiers (1988), observaron un aumento en la cobertura de briofitas epifilas en condiciones de humedad alta.

A pesar de que la alta humedad está asociada a lugares con sombra, esto no significa que las briofitas evaden la luz, por el contrario, las epífilas nunca ocurren en lugares completamente oscuros (o secos) y si la humedad lo permite, pueden crecer en lugares abiertos como en plantaciones de cacao (Rodríguez-Castro, pers. Obs., 2017). Para este estudio, otro de los factores tomados en cuenta para cada micrositio fue la ausencia o presencia de luz. Utilizando la misma prueba de Wilcoxon, se comparó la cobertura en los sitios con luz y sitios con sombra y se obtuvo un valor de $\mathrm{p}<0.05$ ( $\mathrm{W}=569.5$ ), lo que indica que la luz es un factor importante en la cobertura de L. elliptica. Este resultado concuerda con los de Marino \& Salazar (1993) y con la observación Coley et al. (1993), quienes mencionan que en BCI las hepáticas crecen más rápidamente en lugares con alta intensidad de luz comparado con los de sombra; aunque existen efectos tanto positivos como negativos en la colonización y crecimiento de las epífilas en cuanto a los niveles altos de luz (Olarinmoye, 1975). Leptolejeunea elliptica podría ser considerada indicadora de lugares con bastante iluminación, siendo la 
especie que predomina en los claros. En muchas comunidades tropicales del sotobosque, los niveles de luz están entre 0.5 y $5 \%$ a pleno sol (Chazdon \& Fetcher, 1984, Oberbauer et al., 1988); en los sitios de estudio la intensidad de luz fue del 1 al $2 \%$, aunque esta no es una intensidad de luz muy alta hay que mencionar que durante los meses de muestreo se dio la caída de algunos arbustos y algunas de las hojas permanecieron más expuestas que otras y estas fueron los que contaron con una mayor cobertura comparadas con el resto.

Cuadro 4. Temperatura promedio en cada sitio

\begin{tabular}{|l|l|c|}
\hline Estación & Sitio & $\begin{array}{c}\text { Temperatura } \\
\text { promedio }\left(\mathbf{C}^{\circ}\right)\end{array}$ \\
\hline Lluviosa & Húmedo & 25.7 \\
\hline Lluviosa & Menos húmedo & 26.1 \\
\hline Seca & Húmedo & 26.9 \\
\hline Seca & Menos húmedo & 27.0 \\
\hline
\end{tabular}

En cuanto a la temperatura (Cuadro 4), se obtuvo un valor de $\mathrm{p}=0.534$ $(\mathrm{W}=1409)$ lo que indica que no hay diferencias significativas entre los valores de cada micrositio.

La cobertura de L. elliptica también se vio afectada por la precipitación, observándose una disminución (Figuras 5 y 6) durante el mes de noviembre. En este trabajo se observó una leve disminución en la cobertura de L. elliptica en las hojas de $P$. horizontalis, las razones específicas de esta disminución requieren estudios adicionales con censos mucho más seguidos, utilizando solo una especie de arbusto.

Existe evidencia de que los epífilos parecen estar protegidos químicamente, en L. elliptica la presencia de terpenoides pueden beneficiar al huésped al reducir la herbivoría (Chopra \& Kumra, 1988; Mueller \& Wolf-Mueller, 1991). Sin embargo, se observó en $P$. horizontalis herbivoría, lo cual pudiese estar vinculado a la presencia de domacios en sus hojas. Esta variable que, a pesar de no haber sido considerada en este trabajo, se menciona porque la mayoría de las hojas de $P$. horizontalis contaban con mayor superficie foliar con respecto a las hojas de $H$. prunifolius, en muchas se comenzó a observar herbivoría al avanzar los meses de estudio y esto provocó una disminución de su 
área foliar y, por ende, disminución en la colonización y cobertura de la epífila.

Cuadro 5. Estadístico de modelo lineal generalizado

\begin{tabular}{|l|l|l|l|}
\hline & $\begin{array}{l}\text { Desviación } \\
\text { estándar }\end{array}$ & $\mathbf{Z}$ value & $\operatorname{Pr}(>|\mathbf{z}|)$ \\
\hline Humedad & -0.2219 & -1.352 & 0.17625 \\
\hline Luz & -1.5457 & -8.884 & $<2 \mathrm{e}-16^{* * *}$ \\
\hline
\end{tabular}

Signif. Codes: 0

El Modelo Lineal Generalizado (GLM) del Cuadro 5 demuestra que la cantidad de luz es el factor más determinante en la colonización y cobertura de L. elliptica. Para tener un mejor entendimiento sobre el efecto de las condiciones microclimáticas sobre la colonización y cobertura de diferentes especies de briofitas epífilas, es necesario realizar estudios adicionales considerando solo una especie de arbusto y tener mayor control de factores como la luz y humedad, ya que se torna complicado mantener una de las hojas en sombra durante todos los censos y, además, ocurren caídas de árboles exponiéndolas a mayor intensidad de luz. Estos dos factores, humedad y temperatura, tienen gran influencia, pero esto varía según la especie epífila; esto podría significar que si a una especie le beneficia mayor incidencia de luz como a L. elliptica, no tiene que ser lo mismo para otras especies; ocurriendo lo mismo en la humedad.

\section{CONCLUSIONES}

Se observó pocas diferencias en la colonización de L. elliptica sobre las hojas de los arbustos $H$. prunifolius y $P$. horizontalis. Sin embargo, los valores más altos de ramas colonizadoras se observaron en las hojas de ambos arbustos presentes en el micrositio húmedo con luz; pudiéndose considerar entonces a L. elliptica como una especie que se beneficia al crecer en sitios húmedos y con claros. La colonización y cobertura de $L$. elliptica fue mayor en las hojas de los arbustos ubicados en los sitios con luz, lo que sugiere que este último factor es altamente determinante para ambos eventos.

El mayor porcentaje de colonización de L. elliptica no pudo ser establecido con exactitud, ya que no se determinaron diferencias 
significativas en la colonización entre cada uno de los censos. Esto se debe probablemente debido a la colonización por parte de L. elliptica que ocurre de igual forma durante todo el año o quizás, porque este trabajo de seis meses fue muy corto para poder observarlo. Aunque, podemos mencionar que durante el mes de septiembre hubo un número de colonizaciones un poco más elevado en comparación de los otros meses de estudio.

La humedad relativa en los sitios húmedos y secos se mantuvo en un rango de $83.5 \%$ al $97.3 \%$ durante los meses de investigación, por lo que ambos sitios contaban con suficiente humedad relativa para que $L$. elliptica colonizara y cubriera las hojas de ambos arbustos.

\section{AGRADECIMIENTOS}

Al Instituto Smithsonian de Investigaciones Tropicales y su beca Barro Colorado. Al Herbario de la Universidad de Panamá (PMA) por el uso de sus instalaciones. A la Dra. Noris Salazar Allen por el apoyo con la literatura; a las Dras. Anna Mežaka y Katrin Wagner por el apoyo en el trabajo de campo y en el análisis de datos; a Josué Collins y Luisana Mendoza también por el apoyo en el trabajo de campo.74

\section{REFERENCIAS}

Aide, T. M., 1989. The relationship between leaf phenology and herbivory in a tropical understory community. Ph.D. dissertation, Dep, of Biology, Univ, of Utah, Salt Lake City.

Chazdon, R. L. \& N. Fetcher., 1984. Photosynthetic light environments in a lowland tropical rain forest in Costa Rica. Journal of Ecology 72:553-564.

Claver, F. I., 1984. Guía para la elaboración de estudios del medio físico: contenido y metodología. Serie manuales 3. Centro de Estudios de Ordenación del Territorio y Medio Ambiente, Madrid. 572 pp. 
Coley, P.D., T.A. Kursar \& J.L. Machado., 1993. Colonization of tropical rain forest leaves by epiphylls: effects of site and host plant leaf lifetime. Ecology 74(2): 619-623.

Coley, P. D. \& T.A. Kursar., 1996. Causes and consequences of epiphyll colonization. Pp. 337-362 in Mulkey S. S., Chazdon R. L. \& Smith A. P. (eds.). Tropical forest plant ecophysiology. Chapman and Hall, New York.

Chopra, R. N. \& P. K. Kumra., 1988. Biology of bryophytes. John Wiley $\&$ Sons, New York, New York, USA.

De Gracia, J. E., 2002. Composición y distribución de comunidades de hepáticas epífilas en hojas de Hybanthus prunifolius (Schult.) Schulze en la Isla de Barro Colorado. Tesis de Licenciatura. Universidad de Panamá.

Dietrich, W., D. Windsor \& T. Dunne., 1992. Geología, clima e hidrología de la Isla Barro Colorado. En: Leigh, E., S. Rand \& D.M. Windsor., 1992. Ecología de un Bosque Tropical: Ciclos estacionales y Cambios a Largo Plazo. 25-51. Smithsonian Tropical Research Institute. Balboa, Panamá 547 pp.

Frahm, J.P., 1987. Wich factors control the growth of epiphytic bryophytes in tropical rainforest? Symposia Biologica Hungarica 35:639-648.

Fulford, M., B. Crandall \& R. Stotler., 1970. The ecology o fan elfin forest in Puerto Rico. II. The leafy hepaticae of Pico del Oeste, Journal of Arnold Arboretum 51:56-69.

Gradstein S. R., 1997. The taxonomic diversity of epiphyllous bryophytes. Abstracta Botánica. 21(1): 15-19.

Gradstein, S. R. \& A.L. Ilkiu-Borges., 2009. Guide to the plants of Central French Guiana. Part IV. Liverworts and hornworts. Memoirs of the New York Botanical Garden, 76 (4): 1-140.

Lücking, A., 1995. Diversität und Mikrohabitatspräferenzen epiphyller Moose in einem tropischen Regenwald in Costa Rica. PhD thesis, Georg- August-University, Göttingen, Germany. 
Maciel-Silva, A. S., I.F. Marques Valio \& H. Rydin., 2012. Altitude affects the reproductive performance in monoicous and dioicous bryophytes: Examples from a Brazilian Atlantic rainforest. AoB PLANTS pls016doi: 10.1093/aobpla/pls016.

Marino, P.C., 1991. Competition between mosses (Splachnaceae) in patchy habitats. Journal of Ecology. 79 1031-1046.

Marino P. C. \& N. Salazar Allen., 1993. Tropical epiphyllous hepatic communities growing on two species of shrubs in Barro Colorado Island, Panama: The influence of ligth and microsite. Lindbergia, 17: 91-95.

Mueller, U.G. \& B. Wolf-Mueller., 1991. Epiphyll deterrence to the leafcutter ant Atta cephalotes. Oecologia (Berlin) 86: 36-39.

Monge-Najera, J. \& M.A. Blanco., 1995. The influence of leaf characteristics on epiphyllic cover: a test of hypotheses with artificial leaves. Tropical Bryology 11: 5-9.

Oberbauer, S. F., D. B. Clark, D. A. Clark \& M. Quesada., 1988. Crown light environments of saplings of two species of rain forest trees. Oecologia (Berlín) 75:207-212.

Olarinmoye S.O., 1974. Ecology of epiphyllous liverworts: growth in the natural habitats in Western Nigeria. Journal of Bryology, 8: 275-289.

Olarinmoye, S.O., 1975. Ecological studies of epiphyllous liverworts in Western Nigeria. II. Notes on competition and succesional change. Revue Bryologique et Lichénologique 41: 457463.

Pócs, T., 1982. Tropical forest bryophytes. Bryophyte Ecology (ed. A. J. E. Smith) Chapman and Hall, London, pp 333-381.

Pócs, T., 1996. Epiphyllous liverwort diversity at worldwide level and its threat and conservation. Anales Inst. Biol. Univ. Nac. Autón. Mexico, Ser. Bot, 67 (1): 109 - 127. 
Richards, P. W., 1954. Notes on the bryophytes communities of lowland tropical rainforest with species reference to Moraballi Creek, British Guiana. Vegetatio, 5-6, 319-328.

Richards, P.W., 1984. The ecology of tropical forest bryophytes. Pages 1233-1270 in R.M. Schuster, editor. New manual of bryology. Volume 2. Hattori Botanical Laboratory, Nichinan, Japan.

Ruinen, J., 1961. The phyllosphere, an ecological neglected milieu. PlantandSoil15:81-109.

Smith, A. J. E. (1982). Bryophyte Ecology. Chapman and Hall, London.

Sonnleitner, M., S. Dullinger, W. Wanek \& H. Zechmeister., 2009. Microclimatic patterns correlate with the distribution of epiphyllous bryophytes in a tropical lowland rain forest in Costa Rica. Journal of Tropical Ecology, 25: 321-330.

Slack, N. G., 1977. Species diversity and community structure in bryophytes: New York State Studies. New York State Museum. Bulletin 428: 1-70.

Thiers, B. M., 1988. Morphological adaptations of the Jungermanniales (Hepaticae) to the tropical rainforest habitat. Journal of the Hattori Botanical Laboratory 64:5-14.

Toyota, M., Koyama, H. \& Asakawa, Yoshinori., 1997. Volatile components of the liverworts archilejeunea olivaceae, Cheilolejeunea imbricate and Leptolejeunea elliptica. Phytochemistry, Vol. 44, No. 7, pp. $1261-1264$.

Van Reenen, G. B. A., 1987. Altitudinal bryophyte zonation in the Andes of Colombia: a preliminary report. Symposia Biologica Hungarica 35:631-636.

Winkler, S., 1967. Die epiphyllen Moose der Nevelwalder von El Salvador, S. C. Revue Bryologique et Lichenologique, 35, 303-369. 
Wong, M. \& J. Ventocilla., 1987. A day on Barro Colorado Island. Impretex, S. A. Panamá. 93 págs.

Zartman, C. E., H.E.M. Nascimento, K.G. Cagani, L.D.P. Alvarenga \& T. Snäll., 2012. Fine-scale changes in connectivity affect the metapopulation dynamics of a bryophyte confined to ephemeral patches. Journal of Ecology 100: 980-986.

Zartman, C. E., J.A. Amaral, J.N. Figueiredo \& C.S. Dambros., 2015. Drought Impacts Survivorship and Reproductive Strategies of an Epiphyllous Leafy Liverwort in Central Amazonia. Biotropica 47(2): 172-178. 